\title{
Being rich helps - the case of the sdBV KIC 10670103
}

\author{
Jurek Krzesinski and Szymon Bachulski \\ Pedagogical University of Cracow, \\ ul. Podchorazych 2, 30-084 Cracow, Poland \\ email: jk@astro.as.up.krakow.pl
}

\begin{abstract}
We present a study of KIC 10670103, a pulsating hot subdwarf in the Kepler field. By means of Fourier analysis, we investigate periodic signals associated with pulsations. Using asymptotic relationships and rotational multiplets we identify degrees of modes. The Fourier spectrum appears to be rich in $l=1$ and $l=2$ multiplets allowing derivation of a $\sim 90$-day rotation period of the star from rotational splittings. Comparing the identified gravity-mode period spacing pattern with theoretical models we show that KIC 10670103 has to be a thickenvelope sdBV.
\end{abstract}

Keywords. stars: subdwarfs, oscillations, rotation

\section{Introduction}

Pulsating stars are a rich source of knowledge about stellar interiors and evolution. Thanks to asteroseismology, we have the tools to study their internal compositions, envelope masses, rotation and other parameters by interpreting their frequency spectra (FS). In this work, we present the properties of a pulsating hot subdwarf (sdBV) star, KIC 10670103, in the Kepler field (Borucki et al. 2010, Koch et al. 2010, Jenkins et al. 2010).

The sdBVs are extended horizontal branch stars with masses of about $0.5 M_{\odot}$ and effective temperatures between 20000 and $40000 \mathrm{~K}$ (Saffer et al. 1994). Having lost too much of their hydrogen envelope mass prior to the helium flash, they cannot sustain H-shell burning. Therefore, they will not become asymptotic giant branch stars; instead, they will simply cool down as white dwarfs.

The sdBVs come in two brands: short-period (1.5 - 5 minutes) p-mode, V361 Hya-type pulsators (Kilkenny et al. 1997) and long-period (45-120 minutes) g-mode, V1093 Hertype pulsators (Green et al. 2003). There are also a few hybrid sdBV stars known; they show both types of modes simultaneously.

KIC 10670103 is a g-mode pulsator, but has some extreme properties which make it a unique star for seismic analysis. It has the longest observed pulsation periods (up to 4.5 hours) and the lowest effective temperature, $T_{\text {eff }} \approx 20900 \mathrm{~K}$ (Reed et al. 2010, 2011) of all sdBV stars. Using one month of data from the Kepler exploratory phase, Reed et al. (2010) identified 28 modes, which made KIC 10670103 one of the richest pulsators among the sdBV stars. Since then, Kepler has observed KIC 10670103 for over 2.5 years and it is time to see what can be done with 30 months (Q5-Q14) of new data. Figure 1 presents the pulsation pattern of the star, i.e. the FS of 928 days ( $\sim 30$ months) of KIC 10670103 time-series photometry. The pulsation pattern is dominated by two largeamplitude modes with frequencies between 130 and $150 \mu \mathrm{Hz}$. These are responsible for the $\sim 2.5$-day beat present in the light curve of the star. 


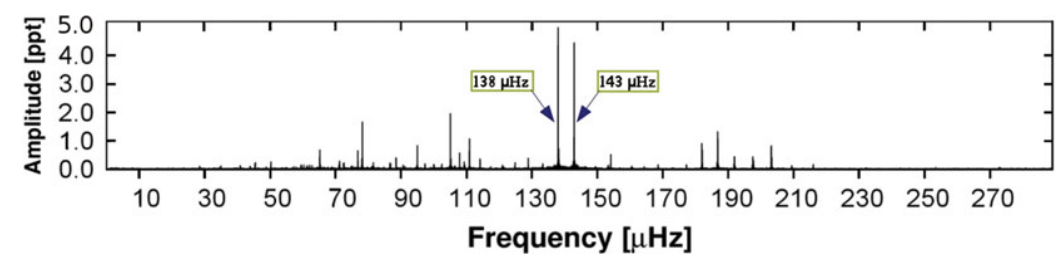

Figure 1. The FS of the 2.5-year Kepler data of KIC 10670103. The pulsation pattern of the star is dominated by two large-amplitude modes (marked with arrows).

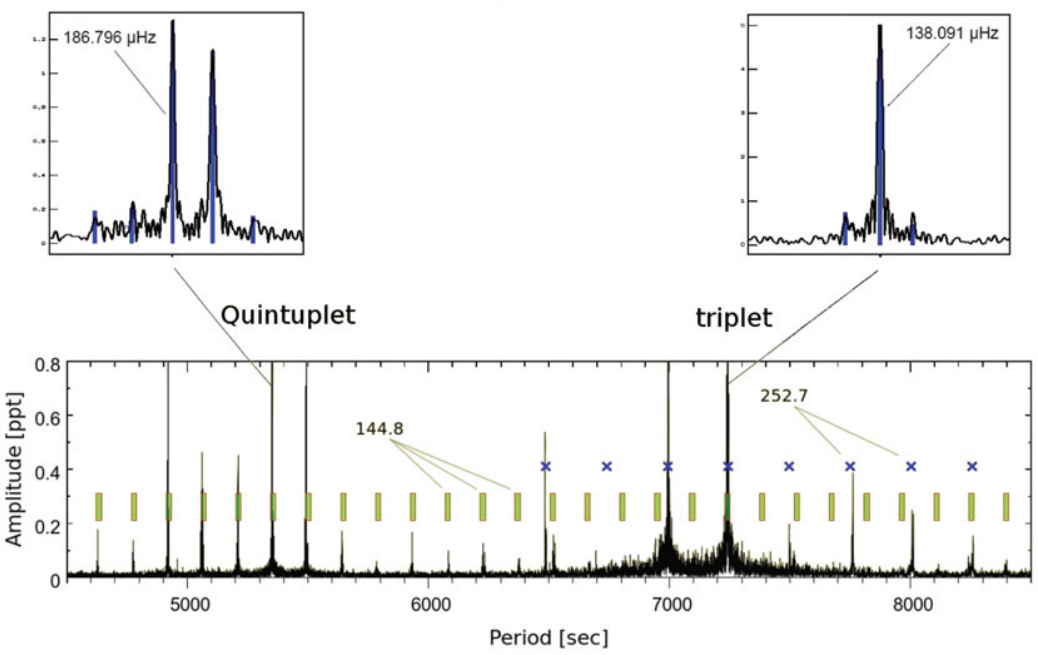

Figure 2. Mode identification from period spacing (at the bottom) and from multiplets (upper part of the figure). Vertical rectangles mark positions of $l=2$ modes while crosses denote $l=1$ modes. The numbers 144.8 and 252.7 denote the period spacings (in s) for these modes.

\section{Mode identification}

Having found the frequencies of the modes, one can perform mode identification in either the period or frequency domains. For g modes, the former allows identification of the degree $l$ using period spacings in the asymptotic limit (Unno et al. 1979, Smeyers \& Tassoul 1987). A neat and brief description of the procedure can be found in Reed et al. (2011).

As one can see in Fig. 2 (bottom panel), there are two distinct spacings in the FS. The shorter, $144.8 \mathrm{~s}$ (marked with a set of vertical rectangles) corresponds to the $l=2$ mode period spacing. The longer one, $252.7 \mathrm{~s}$ (marked by a set of crosses), corresponds to $l=1$ modes. In Fig. 2, some crosses appear at the same positions as rectangles and one may not be able to distinguish between $l=1$ and $l=2$ modes, unless the identification can be done via multiplets.

An example of such a mode can be found near the period of $7250 \mathrm{~s}$. A closer look at its structure (upper right panel of Fig. 2) allows identification of the mode as $l=1$ since a triplet is clearly visible. The decision whether it is indeed a triplet or in fact a partial quintuplet can be made on the basis of the spacings of the components of the multiplet. Multiplets are created when the rotation of a star lifts the azimuthal node degeneracy, and the spacing between multiplet components depends on the angular velocity of the star and on $l$. Therefore, the spacing can be used not only to identify the modes/multiplets, but also to determine the rotation period of the star. In the case of KIC 10670103, the spacing for quintuplets ( $l=2$ modes) is $0.107 \mu \mathrm{Hz}$, while it is $0.069 \mu \mathrm{Hz}$ for triplets 

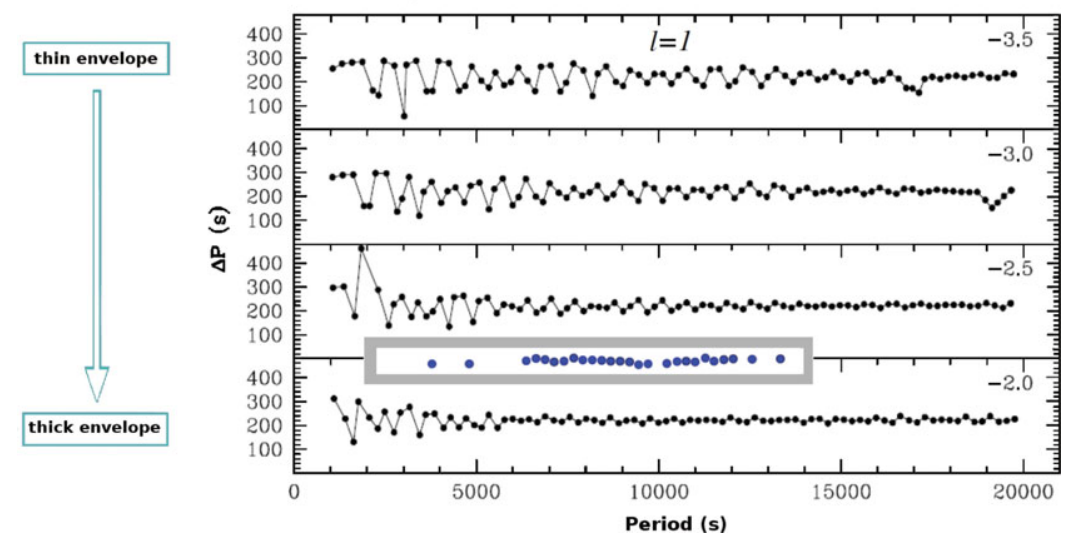

Figure 3. A part of Fig. 4 by Charpinet et al. (2013) presenting the deviations of trapped gravity $l=1$ mode periods from equal period spacing $(\Delta P)$ for different thicknesses of the hydrogen envelope. The envelope mass $\left(\log q(\mathrm{H})=\log M_{\text {env }} / M_{*}\right)$ is shown in the right upper corner of each panel. The rectangle inserted between two bottom panels, with a sequence of solid circles, represents $l=1$ modes identified in this work. The sdBV model with the thickest envelope fits the trapping pattern of KIC 10670103 best.

( $l=1$ modes). The rotation periods derived from the triplet and quintuplet spacings are equal to $\sim 84$ and $\sim 90$ days, respectively.

Using both the period spacing and multiplets, one can double check mode identification whenever possible and prepare a diagram of period spacings vs. period, to look for a gravity mode trapping pattern. It is crucial to identify long continuous chains of modes, since some conclusions about the stellar structure can be made right from the scatter of period spacing diagrams, such as those presented by Charpinet et al. (2013) (their Fig. 4). It appears that the chemical transition between the envelope and mantle of an sdBV star results in a distinct pattern of trapped modes in the period spacing diagrams. As a result, the mean period spacing between consecutive modes of the same degree $l$ is cyclically perturbed. For thin envelopes, the deviation from equal period spacing is severe for all periods. For thick envelopes, mode trapping causes large scatter of period spacings for periods shorter than $5000 \mathrm{~s}$ and becomes small for longer periods, where the period spacing is virtually constant. In Fig. 3 we present a partial set of models from Charpinet et al. (2013) with an inset of the observed g-mode trapping pattern for KIC 10670103. The observed pattern is best fit by the sdBV model with the thickest envelope.

\section{Summary}

Thanks to the long time-series photometry from Kepler, we were able to extract over 300 frequencies from the light curve of KIC 10670103. Using period spacings and multiplet patterns we identified $l=1$ and $l=2$ modes of KIC 10670103 and we used them to determine the stellar rotation period and the mass of its hydrogen envelope. It appears that the star is a very slow rotator with a rotation period of about 90 days. Since some white dwarfs are also slow rotators (Fontaine, these proceedings), slowly rotating sdBs may be an evolutionary link between red giants and slowly rotating white dwarfs.

Comparing our period spacing pattern of $l=1$ (and $l=2$ modes, not shown here) with Charpinet et al. (2013) (their Fig. 3), we conclude that the weak gravity mode trapping observed in KIC 10670103 is due to the thick envelope of the star. The mass of the hydrogen envelope defined as $\log q(\mathrm{H})=\log \left(M_{\mathrm{env}} / M_{*}\right)$ was estimated to be between -2.5 
and -2 . This probably makes KIC 10670103 the star with the most massive hydrogen envelope among all known sdBVs. This conclusion requires verification against a model computed specifically for KIC 10670103 but the grid of models presented by Charpinet et al. (2013) allows for such a preliminary conclusion.

\section{Acknowledgement}

This project was supported by Polish National Science Center grant 2011/03/D/ST9/ 01914.

\section{References}

Borucki, W. J., Koch, D., Basri, G., et al. 2010, Science, 327, 977

Charpinet, S., Van Grootel, V., Brassard, P., Fontaine, G., Green, E. M., \& Randall, S. K. 2013, in: J. Montalbán, A. Noels, \& V. Van Grootel (eds.), Ageing Low Mass Stars: From Red Giants to White Dwarfs, European Physical Journal Web of Conferences, 43, 4005

Green, E. M., Fontaine, G., Reed, M. D., et al. 2003, ApJ, 583, L31

Jenkins, J., Caldwell, D. A., Chandrasekaran, H., et al. 2010, ApJ, 713, L87

Kilkenny, D., Koen, C., O'Donoghue, D., \& Stobie, R. S. 1997, MNRAS, 285, 640

Koch D. G., Borucki, W. J. Basri, G., et al. 2010, ApJ, 713, L79

Reed, M. D., Kawaler, S. D., Østensen, R. H., et al. 2010, MNRAS, 409, 1496

Reed, M. D., Baran, A., Quint, A. C., et al. 2011, MNRAS, 414, 2885

Safer, R. A., Bergeron, P., Koester, D., \& Liebert, J. 1994, ApJ, 432, 351

Smeyers, P. \& Tassoul, M. 1987, ApJS, 65, 429

Unno, W., Osaki, Y., Ando, H., \& Shibahashi, H. 1979, Nonradial Oscillations of Stars (Tokyo: Univ. of Tokyo Press) 